\title{
Demographics, Microbiology and Outcome in Necrotizing Soft Tissue Infections
}

\author{
Chance Witt, MD, Sharmila Dissanaike, MD
}

\begin{abstract}
Background: Necrotizing soft tissue infections (NSTI) are potentially severe infections that have a high morbidity and mortality even with modern medical care. This study examines factors associated with outcomes in patients with NSTI in an academic tertiary care hospital.

Design: This is a retrospective cohort study of patients admitted with NSTI between 2003 and 2008. Baseline demographics and comorbid conditions, laboratory and clinical parameters, timing of surgery, and outcomes, including length of stay and mortality, were compared with univariate analysis; significant factors were then analyzed for their effects on mortality using binary logistic regression analysis.

Results: Sixty-nine patients with NSTI were analyzed; 61\% were men. Diabetes (39\%) was the most common comorbid condition. Most infections (55\%) were polymicrobial. The most common organism in monomicrobial infections was Staphylococcus aureus, and $50 \%$ of these isolates were methicillin resistant. Nine patients (13\%) required amputation. Mortality was $20 \%$, and the most significant predictor of mortality was a higher respiratory rate on admission $(p=0.02)$.
\end{abstract}

Conclusion: Patients in this series frequently had diabetes, usually had polymicrobial infections, and had a $20 \%$ mortality rate.

Key words: necrotizing soft tissue infection (NSTI), necrotizing fasciitis, gangrene, Staphylococcus aureus

\section{INTRODUCTION}

Necrotizing soft tissue infections (NSTI) are rapidly spreading necrotizing infections of the dermis, subcutaneous tissue, fascia, or muscle. In contrast, cellulitis involves superficial skin structures, and abscesses are localized contained infections. The term

Corresponding author: Sharmila Dissanaike, MD. Contact Information: E-mail: sharmila.dissanaike@ ttuhsc.edu. 3601 4th St, MS 8312, Lubbock, TX 79430.

DOI: $10.12746 /$ swrccc2013.0101.003
NSTI organizes infections which might be defined by several classification systems, such as area of the body (e.g., Fournier's gangrene) or layer involved (e.g., necrotizing fasciitis), into one clinical syndrome which requires both medical and surgical treatment. NSTI is quite uncommon, but most clinicians will see at least one during their career. ${ }^{1}$ The incidence appears to be increasing nationwide for unclear reasons, and the mortality remains high (14 -34\%) even with modern care $^{1-4}$.

The current standard of care for treatment includes prompt identification, early surgical debridement, antibiotic therapy, and supportive care ${ }^{1-6}$. 
Early surgical debridement significantly improves survival; ${ }^{1-3}$ therefore, prompt identification and treatment of NSTI represents an important area of study. Due to the low prevalence, however, NSTI studies have small patient numbers and often have different patient populations. Both these factors make it important to analyze as many groups as possible to identify factors that help in early diagnosis of NSTI and identify patients with a higher likelihood of death. Identification of factors that impact mortality can also serve to identify high-risk patients who might be suitable candidates for trials of experimental therapies, such as plasmapheresis or hyperbaric oxygen. We wanted to compare the differences between patients who survived NSTI and those who did not survive.

\section{Methods}

Patients discharged from the University Medical Center, Lubbock, TX, between 2003 and 2008 with a discharge diagnosis of NSTI were identified using ICD-9 codes 785 and 728. A list of patients was also obtained from the Surgery Department's billing office to cross check records and reduce the possibility of missed documents. The diagnosis was then confirmed by scanning each patient's medical record for documentation of NSTI or a related diagnosis (e.g., Fournier's gangrene, clostridial myonecrosis, and necrotizing fasciitis) by an attending physician. Patients with diagnoses of abscess and cellulitis alone were excluded.

A retrospective review was performed, including demographic information, initial vital signs, laboratory values, microbiology, number of operations, time to diagnosis and time to first operation, admitting service, clinical course, and outcome. Univariate analysis was performed using Student's independent samples t-tests for continuous variables and chi-square test for analysis of proportions. Fisher's exact test was used instead of chi square where any category had fewer than five cases.

All variables significant at $p<0.1$ were then included in a binary logistic regression model with mortality as the outcome using a forced entry method.
Sex and time to surgery were forced into the final model. Variables demonstrating significant collinearity were replaced with one variable (e.g., admission $\mathrm{CO}_{2}$ and respiratory rate, systolic and diastolic blood pressure) based on clinical relevance. The omnibus test of model coefficients and Hosmer-Lemeshow goodness of fit tests were used to assess the performance of the model. Odds ratios (OR) are reported with a $95 \%$ confidence interval $(\mathrm{Cl})$ as well as $p$ values. A value of $p<0.05$ was considered statistically significant. SPSS ver.13.0 (Chicago, IL) was used for all analyses.

\section{RESULTS}

Sixty-nine patients were included with an average age of 50 years (range, 10-92). The racial distribution included $44 \%$ Hispanics $(n=30)$, 46\% Caucasians $(n=32)$, and $10 \%$ Blacks $(n=7) ; 61 \%$ were men. Fourteen patients $(20 \%)$ died, and nine patients $(13 \%)$, including three who died, required an amputation. Table 1 shows the demographic and clinical care variables in survivors and non-survivors. Diabetes was the most common pre-existing condition. Age was the only factor significantly different $(p=0.01)$ between the two groups. All patients underwent surgery, averaging two to three operations each. Mean time to diagnosis and first operation were between one to two days.

Vital signs and lab values taken at admission are shown in Table 2. Respiratory rates on admission were significantly higher in those patients who did not survive (22 breaths per minute vs. 19 breaths per minute, $p=0.01)$. Blood pressures were lower in non-survivors $(p=0.16)$. Higher blood urea nitrogen levels predicted mortality $(p=0.001)$. In multivariate analysis, an elevated respiratory rate on admission remained the single most significant predictor for mortality (OR $0.54,95 \% \mathrm{Cl} 0.325-0.906$ for survival, $p=0.02)$. Older age $(p=0.058)$ and increased serum creatinine levels $(p=0.052)$ were more frequent in non survivors (Table 3 ). The logistic regression model had a highly significant overall coefficient $(p=0.001$ with a chi square of 26.5 and 8 degrees of freedom) and satisfactory goodness of fit (Hosmer-Lemeshow test: chi square of 4.0, $p=0.855$ ). 
Table 1 Demographic and Patient Care Variables, Univariate Analysis, Survival Versus Non-survival

\begin{tabular}{|r|c|c|c|}
\hline Characteristic & Survival & Non-Survival & p Value \\
\hline Age (years) & 47 & 60 & 0.0108 \\
\hline Male & $35(51 \%)$ & $7(50 \%)$ & 0.1646 \\
\hline Diabetes & 23 & & 0.1672 \\
\hline Hypertension & 16 & 4 & 0.1545 \\
\hline Heart Disease & 6 & 4 & 0.3471 \\
\hline Alcohol Use & 11 & 1 & 0.6962 \\
\hline Tobacco Use & 12 & 2 & 0.4298 \\
\hline IV Drug Use & 8 & 3 & 0.532 \\
\hline Patient Care & & & 0.4438 \\
\hline Time to Diagnosis (days) & 1.19 & 0.75 & 0.3720 \\
\hline Time to Surgery (days) & 1.81 & 1.00 & 0.5013 \\
\hline Number of operations & 2.80 & 2.42 & \\
\hline & & & \\
\hline
\end{tabular}

Table 2 Vital Signs and Lab Values on Admission, Univariate Analysis, Survival Versus Non-survival

\begin{tabular}{|c|c|c|c|}
\hline Characteristic & Survival & Non-Survival & p Value \\
\hline Vital Signs & & & \\
\hline Temperature $\left({ }^{\circ} \mathrm{F}\right)$ & 99.1 & 98.5 & 0.3926 \\
\hline Systolic BP $(\mathrm{mmHg})$ & 126 & 109 & 0.0755 \\
\hline Diastolic BP $(\mathrm{mmHg})$ & 71 & 61 & 0.0805 \\
\hline Heart Rate & 102 & 95 & 0.3198 \\
\hline Respiratory Rate & 19 & 22 & 0.0165 \\
\hline Oxygen Saturation (\%) & 97 & 96 & 0.1754 \\
\hline
\end{tabular}


Table 2 Vital Signs and Lab Values on Admission, Univariate Analysis, Survival Versus Non-survival (continued)

\begin{tabular}{|c|c|c|c|}
\hline Characteristic & Survival & Non-Survival & p Value \\
\hline \multicolumn{4}{|l|}{ Lab Values } \\
\hline Sodium (mEq/L) & 134 & 137 & 0.1987 \\
\hline Potassium (mEq/L) & 3.9 & 4.1 & 0.2877 \\
\hline Chloride (mEq/L) & 102 & 107 & 0.1360 \\
\hline Bicarbonate (mEq/L) & 18 & 12 & 0.3041 \\
\hline BUN (mg/dL) & 19 & 45 & 0.0101 \\
\hline Creatinine (mg/dL) & 0.9 & 2.6 & 0.0621 \\
\hline Glucose (mg/dL) & 192 & 132 & 0.1594 \\
\hline Calcium (mg/dL) & 8.2 & 8.4 & 0.5326 \\
\hline CRP (mg/L) & 20.3 & 17.7 & 0.7037 \\
\hline Prealbumin (mg/dL) & 9.1 & 8.1 & 0.7023 \\
\hline WBC count & 17.4 & 14.2 & 0.2537 \\
\hline Hemoglobin (g/dL) & 11.7 & 11.9 & 0.7951 \\
\hline Hematocrit (\%) & 34.6 & 35.8 & 0.5950 \\
\hline Platelet count & 312 & 256 & 0.2657 \\
\hline
\end{tabular}

BP, blood pressure; BUN, blood urea nitrogen; CRP, C-reactive protein; WBC, white blood cell count

Table 3 Results of Binary Logistic Regression Analysis, with Survival as Outcome

\begin{tabular}{|l|c|c|c|c|}
\hline \multirow{2}{*}{ Variable } & \multirow{2}{*}{$\mathrm{p}$ Value } & \multirow{2}{*}{ Odds Ratio } & \multicolumn{2}{c|}{$\begin{array}{c}\text { 95\% C.I. for Odds } \\
\text { Ratio }\end{array}$} \\
\cline { 3 - 5 } & & & Lower & Upper \\
\hline Age & .058 & .900 & .808 & 1.004 \\
\hline Systolic BP & .656 & .982 & .909 & 1.062 \\
\hline Respiratory Rate & .020 & .543 & .325 & .906 \\
\hline Creatinine & .052 & .054 & .003 & 1.022 \\
\hline Sex & .147 & .145 & .011 & 1.970 \\
\hline Time to Surgery & .841 & 1.075 & .532 & 2.170 \\
\hline
\end{tabular}


Table 4 Wound Culture Results

\begin{tabular}{|l|c|}
\hline \multicolumn{1}{|c|}{ Type of Infection } & Number of Positive Cultures \\
\hline Polymicrobial & 37 \\
\hline No Growth & 6 \\
\hline Monomicrobial & 24 \\
\hline Staphylococcus aureus & 15 \\
\hline Methicillin-sensitive & 8 \\
\hline Methicillin-resistant & 7 \\
\hline Streptococcus pyogenes & 4 \\
\hline Streptococcus agalactiae & 2 \\
\hline Enterococcus faecalis & 1 \\
\hline Group G Streptococcus & 1 \\
\hline Acinetobacter & 1 \\
\hline
\end{tabular}

More than half of the infections were Type 2 or polymicrobial (Table 4). Twenty-four monomicrobial (Type 1) infections included fifteen with $S$. aureus (half of these being methicillin-resistant). The remaining infections were mostly attributed to Streptococcus species. There were no clostridial or fungal infections. Most patients had their infections localized to single body areas; $16(23.8 \%)$ had infection in more than one area. The most common locations were the leg in 34 patients $(50.7 \%)$, perineum in 16 patients (23.8\%), arm in $15(22.4 \%)$, abdomen in $12(17.9 \%)$, buttocks in six $(9 \%)$, thorax in four $(6 \%)$, and head in one $(1.5 \%)$.

Internal Medicine services $(n=19)$ and Surgery services $(n=41)$ admitted the majority of patients; the departments of Family Medicine $(n=3)$, Orthopedics $(n=3)$, Pediatrics $(n=2)$ and Obstetrics and Gynecology $(n=1)$ admitted the rest. Twenty-eight patients $(41 \%)$ were transferred from other hospitals, and the majority $(24,86 \%)$ was admitted to the Surgery service. There were no differences in demographics or comorbidities between transfers and the rest of the cohort. The patients who were transferred did have lower blood glucose levels on admission (112 vs. 216, $p=.003)$. Several transfer patients had already had initial operations at the outside hospitals and were transferred primarily for reconstruction; these patients were excluded from subsequent analyses of time to diagnosis and time to surgery. Transferred patients had significantly longer lengths of stay (19.8 vs. 14.8 days, $p<.0005)$ but no differences in mortality.

\section{Discussion}

This study found that an elevated respiratory rate on admission is an independent predictor of death in NSTI patients. Surprisingly, this proved more significant than age, renal failure, and time to surgery. A high respiratory rate is not specifically mentioned in previous studies. However, it is one of the diagnostic criteria for the systemic inflammatory response syndrome and likely reflects increased illness severity. The absolute difference in mean values between survivors and non-survivors was small (19 breaths per 
minute versus 22 breaths per minute) and, therefore, of questionable clinical significance. Nonetheless, we think that this is a useful clinical sign that highlights the importance of physical exam and vital signs in assessing the severity of complex patients.

Older age and renal failure defined by increased creatinine $(2.6 \mathrm{mg} / \mathrm{dl}$ vs. $0.9 \mathrm{mg} / \mathrm{dl})$ and BUN (45 mg/ dl vs. $19 \mathrm{mg} / \mathrm{dl}$ ) were associated with mortality in this analysis but did not reach statistical significance. Previous studies have shown a correlation between both age and renal function and outcome, and it is possible that our small sample size and limited power resulted in a Type II error. ${ }^{5}$ Diabetes is a risk factor for NSTI but does not necessarily portend a worse outcome. $2,7,11$ Our study did not show that it has any effect on mortality.

Wall and coworkers and Wong and coworkers have used laboratory data (a composite of white cell count, hemoglobin, sodium, glucose, creatinine and $\mathrm{C}$ reactive protein) to develop the laboratory risk indicator for necrotizing fasciitis (LRINEC) ${ }^{8,9}$. This tool was initially developed to differentiate NSTI from other soft tissue infections and to aid in earlier diagnosis but not to predict mortality in these patients. Therefore, it is not surprising that the values used for the LRINEC score were similar in our two patient groups.

The types of bacteria identified in this study were similar to those typically seen in NSTI patients ${ }^{10,12}$. The high level of polymicrobial infections (also known as Type 2 as opposed to monomicrobial Type 1) is also quite typical. However, the number of cultures growing Staphylococcal species was somewhat higher than in earlier studies, which have usually identified Streptococcal species as the most common bacteria. This agrees with recent studies that reported that Staphylococcal infections are becoming more common. ${ }^{10,11}$ We had no clostridial or fungal infections in our patients.

This study has several limitations. First, the study size was small and, therefore, limited in power. However, given the rarity of NSTI, its size is comparable to other literature in this field. Second, the recording of time to diagnosis and time to surgery was in days and not in hours. This introduces a lack of precision in these data; we did attempt to obtain more accurate timelines but were limited by gaps in the medical records. However, time to diagnosis and operation did not appear to affect mortality, and more precision is not likely to have changed that result. We need more studies to identify factors that differentiate necrotizing soft tissue infections from less serious infections to hasten diagnosis and treatment. We also need to identify factors that predict increased mortality in patients with NSTI. The importance of this information will potentially increase as we develop more specialized experimental therapies.

\section{Key PoInts}

1. Diabetes is a common predisposing factor for NSTI in this Southwestern US population.

2. Polymicrobial infections are the most common type; monomicrobial infections usually involve Staphy lococcal or Streptococcal isolates.

3. Patients presenting with signs of sepsis syndrome associated with an elevated respiratory rate appear to have higher mortality.

4. The mortality rate remains high even with early surgery and the use of broad spectrum antibiotics. 


\section{REFERENCES}

1. Boyer A, Vargas F, Coste F, et al. Influence of surgical treatment timing on mortality from necrotizing soft tissue infections requiring intensive care management. Intensive Care Med 2009; 35:847-53.

2. Endorf FW, Klein MB, Mack CD, Jurkovich GJ, Rivara FP. Necrotizing soft-tissue infections: differences in patients treated at burn centers and non-burn centers. J Burn Care Res. 2008 Nov-Dec; 29(6):933-8.

3. McHenry CR, Piotrowski JJ, Petrinic D, Malangoni MA. Determinants of mortality for necrotizing soft-tissue infections. Ann Surg 1995; 221:558-63.

4. Anaya DA, McMahon K, Nathens AB, et al. Predictors of mortality and limb loss in necrotizing soft tissue infections. Arch Surg 2005; 140:151-7.

5. Elliott DC, Kufera JA, Myers RA. Necrotizing soft tissue infections. Risk factors for mortality and strategies for management. Ann Surg 1996; 224:672-683.

6. Tillou A, St Hill CR, Brown C, Velmahos G. Necrotizing soft tissue infections: improved outcomes with modern care. Am Surg 2004; 70:841-4.

7. Wong $\mathrm{CH}$, Chang HC, Pasupathy S, et al. Necrotizing fasciitis: clinical presentation, microbiology, and determinants of mortality. J Bone Joint Surg Am 2003; 85:1454-60.

8. Wall DB, de Virgilio C, Black S, Klein SR. Objective criteria may assist in distinguishing necrotizing fasciitis from non-necrotizing soft tissue infection. Am J Surg 2000; 179:17-21.

9. Wong $\mathrm{CH}$, Khin LW, Heng KS, et al. The LRINEC (laboratory risk indicator for necrotizing fasciitis) score: a tool for distinguishing necrotizing fasciitis from other soft tissue infections. Crit Care Med 2004; 32:1535-41.

10. Eke N. Fournier's gangrene: a review of 1726 cases. Br J Surg 2000; 87:718-28.

11. Kao LS, Knight MT, Lally KP, Mercer DW. The impact of diabetes in patients with necrotizing soft tissue infections. Surg Infect 2005; 6:427-38.

12. Mills MK, Faraklas I, Davis C, et al. Outcomes from treatment of necrotizing soft-tissue infections: results from the National Surgical Quality Improvement Program database. Am J Surg 2010; 200:790-6. 